\title{
REAL-TIME SEGMENTATION OF 4D ULTRASOUND BY ACTIVE GEOMETRIC FUNCTIONS
}

\author{
Qi Duan ${ }^{1}$, Elsa D. Angelini ${ }^{2}$, Shunichi Homma ${ }^{3}$, Andrew F. Laine ${ }^{1}$ \\ ${ }^{1}$ Department of Biomedical Engineering, Columbia University, New York, NY, USA \\ ${ }^{2}$ Institut Telecom, Telecom-ParisTech, CNRS LTCI, Paris, France \\ ${ }^{3}$ Department of Medicine, Columbia University, New York, NY, USA
}

\begin{abstract}
Four-dimensional ultrasound based on matrix phased array transducers can capture the complex 4D cardiac motion in a complete and real-time fashion. However, the large amount of information residing in 4D ultrasound scans and novel applications under interventional settings pose a big challenge in efficiency for workflow and computer-aided diagnostic algorithms such as segmentation. In this context, a novel formulation framework of the minimal surface problem, called Active Geometric Functions (AGF), is proposed to reach truly real-time performance in segmenting 4D ultrasound data. A specific instance of AGF based on finite element modeling and Hermite surface descriptors was implemented and evaluated on $354 \mathrm{D}$ ultrasound data sets with a total of 425 time frames. Quantitative comparison to manual tracing showed that the proposed method provides LV contours close to manual segmentation and that the discrepancy was comparable to inter-observer tracing variability. The ability of such realtime segmentation will not only facilitate the diagnoses and workflow, but also enables novel applications such as interventional guidance and interactive image acquisition with online segmentation.
\end{abstract}

Index Terms - Image segmentation, Active Geometric Functions, real-time segmentation, deformable model

\section{INTRODUCTION}

Ultrasound is the cardiac screening modality with the highest temporal resolution, but its use remains limited to two-dimensions in most hospitals and clinical centers. Development of 4D (real-time 3D or RT3D) volumetric echocardiography started in the 1990s [1-3], with 4D ultrasound probes based on matrix phased arrays. Since RT3D ultrasound acquires volumetric ultrasound sequences with fairly high temporal resolution using a fixed-positioned transducer, it can capture complex 3D cardiac motion. With recent advances in $3 \mathrm{D}$ and $4 \mathrm{D}$ imaging techniques towards real-time imaging, the amount of data is becoming prohibitively overwhelming for analysis and workflow. Manual tracing of these large data sets is tedious and impractical in clinical setting.
In this context, automated or semi-automated segmentation methods have been proposed to leverage human efforts involved in the segmentation task. Based on the mathematical foundation of each method, segmentation approaches can be roughly divided into several classes: classification (e.g. thresholding, k-means), region growing (such as fuzzy connectedness [4]), deformable models (e.g. snake [5], level set [6-9]), active shape [10] and active appearance models [11], and stochastic methods (Markov random field [12], graph cut [13]). Hybrid methods [14] combining different existing methods were also proposed. Among segmentation methods, deformable models are still widely used in medical image analysis, especially for cardiac imaging.

The first deformable model parametric formulation was proposed by Kass, et al in 1987 [5]. In 1998, Xu et al [15] proposed the Gradient Vector Flow, or GVF, to overcome several drawbacks in the original snake framework. In order to handle topological changes, especially in $3 \mathrm{D}$, in the late 1990s, Sethian, et al. [16] proposed level set framework by utilizing level set functions with higher dimensionality than the data. In 2001, Chan and Vese [17] proposed their famous "active contour without edges". The driving forces were derived via energy minimization of the Mumford-Shah segmentation functional [18]. Their method has been widely used in ultrasound segmentation [19], brain segmentation [20], and many other applications. However, the introduction of level set functions implicitly increased the number of parameters of the surface model, which increases the demand for computational power. Although many optimization modifications such as narrowbanding or fast marching schemes were proposed, generally speaking, level-set framework is still a relatively "slow" approach, especially for 3D or 4D data.

As imaging technology evolves, demands for real-time feedback also increases, mostly for interventional imaging and minimally-invasive surgery. In this context, a new framework called Active Geometric Functions (or AGF) is proposed in this paper to push the limits towards real-time segmentation.

\section{METHODOLOGY}

\subsection{General Active Geometric Functions Framework}




\subsubsection{Interface Representation}

In all deformable model methods, interface representation is fundamental. Mathematically, there are two ways to represent the interface:

- Explicit representation: that is representing the surface by explicitly listing the coordinates of the boundary points (i.e. a parametric representation). This is the representation that original snakes [21] used;

- Implicit representation: that is representing the surface by embedding the boundary as the iso-value curves of some function $f$ called the representation function. Level set functions [6-9] are a good example by embedding the interface as the zero level-set of a distance function.

\subsubsection{Geometric Function}

Most of the recent efforts in segmentation based on implicit interface representation have focused on the level-set framework, given its advantages for topological changes and feasibility to represent convoluted surfaces. As mentioned above, level set functions add one extra dimension beyond the dimensionality of the image data. For example, to represent a surface in 3D space, the level set function corresponding to the surface will be a tri-variate function. For comparison, original parametric deformable models only required a list of point coordinates in 3D. For level set, this extra dimension brings various benefits as well as additional computation load, which may degrade computational efficiency.

By looking the opposite way of level set frameworks, it is very natural to think of dimensionality reduction in surface representation to reduce the computational complexity. Using terminology of interface representation, we are looking for a representation function which has fewer dimensions than the image data, i.e. using a 2D function to represent a $3 \mathrm{D}$ surface in space. We call such function a geometric function.

Mathematically, in $N$ dimensional space, we can define a geometric function $g: \mathbb{R}^{\mathrm{N}-1} \rightarrow \mathbb{R}$ as a special set of functions representing one of the coordinates constrained by the others. Without losing generality, we can assume that this special coordinate is $x_{0}$ and the other coordinates are $x_{1}$ to $x_{N-1}$. That is:

$$
x_{0}=g\left(x_{1}, \ldots x_{N-1}\right) .
$$

The corresponding representation function $f$ is defined as:

$$
f=x_{0}-g\left(x_{1}, \ldots x_{N-1}\right) \text {. }
$$

So that the corresponding boundary is the zero-value curve of the function $f$, i.e. $f=0$.

\subsubsection{Driving Forces}

Similar to other deformable models, we adopted a variational framework in deriving the driving forces. For example, we can use the Mumford-Shah segmentation energy functional:

$$
E(F, \vec{C})=\beta \int_{\Omega \backslash \bar{C}}(F-G)^{2} d V+\alpha \int_{\Omega \mid \bar{C}}|\nabla F|^{2} d V+\gamma \oint_{\bar{C}} d s
$$

in which $\vec{C}$ denotes the smoothed and closed segmented interface, $G$ represents the observed image data, $F$ is a piecewise smoothed approximation of $G$ with discontinuities only along $\vec{C}$, and $\Omega$ denotes the image domain. Given the flexibility of variational frameworks, other segmentation energy functionals may also be easily adopted.

For the segmentation of an $\mathrm{N}$-dimensional image data set, the Active Geometric Functions framework will solve an (N-1)-dimensional variational problem; explicit representation will solve an $\mathrm{N}$-dimensional problem; and the level set framework will solve an $(\mathrm{N}+1)$-dimensional variational problem. It is obvious that AGF framework has advantages in dimensionality reduction when compared with the other two deformable models formulations, at the cost of some flexibility given the assumption of 1 versus $(\mathrm{N}-1)$ coordinate mapping. However, such surface mapping can be further modified via the combination with finite element models. In addition, for typical medical applications, biological surfaces are relatively smooth and well represented with relatively simple geometric functions.

\subsection{D Ultrasound Segmentation}

\subsubsection{Geometric Function Setup}

AGF is a generic framework. The actual geometric function is not necessarily defined on Cartesian bases. Any spatial basis can be chosen for the purpose of efficiency in surface representation. In cardiac applications, given the ellipsoidal shape of the left ventricle, usually spherical coordinate system [22] or prolate spheroidal coordinates system [23] can be used to exploit the shape prior knowledge. Generally in 3D space a geometric function can be described through an equation $v_{0}=g\left(v_{1}, v_{2}\right)$ with coordinates $\left(v_{0}, v_{1}, v_{2}\right)$. $\left(v_{0}, v_{1}, v_{2}\right)$ can be $(r, \theta, z)$ in cylindrical coordinate systems, $(r, \theta, \phi)$ in spherical coordinate systems, and $(\lambda, \theta, \mu)$ in prolate spheroidal coordinate systems. In this paper, although prolate spheroidal coordinate systems proposed by Hunter [24] was used, all formulations were expressed in generic form and free of change in coordinate system.

Another benefit of AGF is that it does not require using a single function to represent the entire surface. Piecewise smooth functions building on conceptual patches can be adopted for accuracy and flexibility. Specifically, in this paper, geometric functions described by a conceptual finite element model utilizing cubic Hermite polynomials as geometric function basis was used to efficiently represent the convoluted endocardial surface. The entire endocardial surface was represented by geometric functions built on a "mesh" composed by $8 \times 8$ conceptual patches. Given the dimensionality reduction of the geometric function representation, on each conceptual patch, a 2D cubic geometric function was defined, using cubic Hermite polynomials as basis functions. In $1 \mathrm{D}$, given a normalized 
coordinate $\xi \in[0,1]$ defined on a $1 \mathrm{D}$ patch, there were four cubic Hermite basis functions:

$$
\begin{aligned}
& \mathrm{H}_{0}^{0}(\xi)=1-3 \xi^{2}+2 \xi^{3}, \mathrm{H}_{0}^{1}(\xi)=\xi(\xi-1)^{2} \\
& \mathrm{H}_{1}^{0}(\xi)=\xi^{2}(3-2 \xi), \mathrm{H}_{1}^{1}(\xi)=\xi^{2}(\xi-1)
\end{aligned}
$$

On a $2 \mathrm{D}$ finite element patch, these four basis functions were associated with each local coordinate direction $\left(\xi_{1}\right.$ or $\xi_{2}$ ), which generated a total of $162 \mathrm{D}$ basis functions. On each node of a four-node patch, there were four Hermite coefficients $H^{i}, i=1,2,3,4$, controlling the weights of each basis function. Figure 1 shows an example using $3^{\text {rd }}$ order Hermite polynomials to represent a convoluted 3D surface in 3D space using a single 2D finite element patch. For comparison, if linear quadrilateral patches were used, about 100 linear patches would be required to represent the same surface with comparable accuracy. For this reason, Hermite polynomials are widely used in cardiac biomechanics studies for surface representation [25-27]. A simple $8 \times 8$ finite element model (FEM) with intrinsic $\mathrm{C}^{1}$ continuity can sufficiently represent the geometry of the endocardium [26, 27]. In our implementation, this $8 \times 8$ convention was followed,

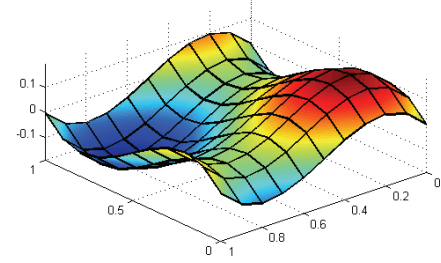

Figure 1: Illustration of cubic Hermite surface representation: a single patch with cubic Hermite representation can efficiently characterize a convoluted surface as shown in color, whereas 100 linear quadrilateral patches would be needed to achieve similar accuracy.

\subsubsection{Energy Minimization}

Following the same rationale used in the Chan and Vese level set [17] framework, the energy in equation (3) can be minimized via a Newton Downhill method:

$$
H^{i, t+d t}=H^{i, t}-d t \frac{\partial E}{\partial H^{i}},
$$

with $d t$ representing the artificial time step in numerical iterations. It has been shown in [23] that for binary segmentation problem, into two partitions $\left\{\Omega^{i}\right\}_{i=1,2}$ of the image domain $\Omega, \frac{\partial E}{\partial H^{i}}$ has the surface integral form of

$$
\frac{\partial E}{\partial H^{i}}=\int_{v_{1}, v_{2}}\left(2 f^{i}\left(u-\frac{c_{1}+c_{2}}{2}\right)\left(c_{2}-c_{1}\right) V+v \frac{\partial A}{\partial H^{i}}\right) d v_{1} d v_{2},
$$

with $f^{i}$ representing the surface coefficient with respect to the basis function values at current surface location, $c_{l}$ and $c_{2}$ representing the average intensity values in the two partitions, $V$ representing the scaling factor due to coordinate transformation and $A$ representing the surface area. Details on the computation of this term can be found in [23]. The second part in the integration is a curvature term, which is composed by two non-linear terms involving $H^{i}$ and scaling factors. Due to the intrinsic continuity in the Hermite representation, contribution from this term was usually very small. For this reason, and for costeffectiveness, this terms can be either suppressed as proposed in [23] or replaced by an equivalent linear term [22] derived from minimizing curvature instead of the surface area as in the original Chan and Vese framework.

\section{RESULTS}

The proposed method was tested on $354 \mathrm{D}$ data sets containing 425 frames, acquired by a Philips $($ iE33 ultrasound machine during five separate canine experiments, with various degrees of induced ischemia as well as controlled stages. Each data set contained 10-15 volumetric frames, depending on the heart rate. Each volume was about $200 \times 200 \times 200$ in matrix size with pixel size of $0.8 \mathrm{~mm}$ in each dimension. For quantitative comparison purpose, endocardial borders for all data sets were manually traced by an experienced expert with a computer-aided interface. Eleven data sets were also traced by two other experts to estimate the inter-observer variability. Distances between two surfaces served as quantitative metrics to describe surface discrepancy.

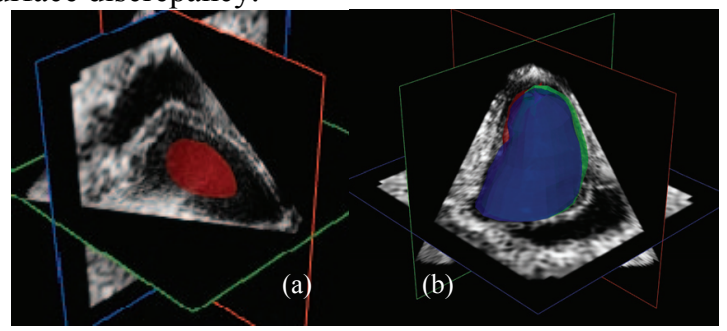

Figure 2: (a) Automatic initialization of the LV surface with an ellipspoid positioned at the center of the volume; (b) overlaid segmentation results from AGF (red), one expert (green), and the other expert (blue). All three surfaces were very close to each other.

As shown in Figure 2a, all 425 segmentation experiments were initialized as a small ellipsoid (defined as an isosurface in prolate spheroidal coordinates) at the center of the image volume and aligned with the vertical direction of the image data, without using any prior knowledge. The segmentation was fully automated without any manual modification. A sample frame overlaying segmentation from AGF (red) and manual tracings from two experts (green and blue) is shown in Figure 2b. All three surfaces were very close to each other. This observation was confirmed by quantitative validation. On all 425 frames, the mean distance of AGF segmentation to manual tracing was 4.00 $\mathrm{mm}$ (about 3 times the pixel diagonal dimension) with a standard deviation of $3.23 \mathrm{~mm}$; the mean distance between two manual tracings was $4.23 \mathrm{~mm}$ with a standard deviation of $3.26 \mathrm{~mm}$.

On average, it took AGF $32.9 \mathrm{~ms}$ to converge for one $3 \mathrm{D}$ frame, on a regular Pentium $2.0 \mathrm{GHz}$ PC running Redhat 
Linux, enabling a potentially 33 fps segmentation rate. Note that this rate is faster than the actual imaging acquisition rate (up to $25 \mathrm{~Hz}$ ), suggesting $\mathrm{AGF}$ could enable online segmentation.

\section{CONCLUSION}

Active Geometric Function (AGF) was presented as a new framework for deformable models. It offers significant advantages in computational efficiency, derived from a dimensionality reduction in the interface representation. It utilized geometric function representation, enabling easy partition of the image data as well as straightforward quantitative segmentation comparison. Moreover, besides providing numerical solutions to the desired optimal interface positioning, like a level set framework, AGF can use closed form expressions as well, to achieve even better efficiency and accuracy. The continuous form of interface function can also benefit downstream analysis based on shape or other information from the interface. The performance of AGF was illustrated in segmenting 4D ultrasound data, with comparable performance to manual tracing and very fast segmentation at $30 \mathrm{~ms}$ per $3 \mathrm{D}$ frame. The ability of real-time segmentation will not only facilitate diagnosis, but also enable novel applications such as interventional guidance and interactive image acquisition with online segmentation.

\section{REFERENCES}

[1] O. T. V. Ramm and S. W. Smith, "Real time volumetric ultrasound imaging system," Journal of Digital Imaging, 3, pp. 261-266, 1990.

[2] S. W. Smith, H. G. Pavy, and O. T. Ramm, "High-speed ultrasound volumetric imaging system. I: Transducer design and beam steering," IEEE Transactions on Ultrasound, 38, pp. 100108, March 1991.

[3] S. W. Smith, H. G. Pavy, and O. T. Ramm, "High-speed ultrasound volumetric imaging system. II: Parallel processing and image display," IEEE Transactions on Ultrasound, 38, pp. 109115, March 1991.

[4] J. K. Udupa, W. L., S. Samarasekera, et al., "Multiple sclerosis lesion quantification using fuzzy Connectedness principles," IEEE Transactions in Medical Imaging, 16, pp. 598-609, 1997.

[5] M. Kass, A. Witkin, and D. Terzopoulos, "Snakes: Active contour models," International Journal of Computer Vision, 1, pp. 321-331, 1987.

[6] J. Sethian, Level set methods and fast marching methods, 2 ed. 3. Cambridge: Cambridge University Press, 1999.

[7] S. Osher and R. Fedkiw, Level set methods and dynamic implicit surfaces 153. New York: Springer, 2003.

[8] T. F. Chan and L. A. Vese, "Active contours without edges," IEEE Transactions on Image Processing, 10, pp. 266 - 277, 2001.

[9] E. Angelini, S. Homma, G. Pearson, et al., "Segmentation of real-time three-dimensional ultrasound for quantification of ventricular function: a clinical study on right and left ventricles," Ultrasound in Medicine and Biology, 31, pp. 1143-1158, 2005.

[10] T. F. Cootes, C. J. Taylor, D. H. Cooper, et al., "Active Shape Models-Their Training and Application," Computer Vision and Image Understanding, 61, pp. 38-59, 1995.
[11] T. F. Cootes, G. J. Edwards, and C. J. Taylor, "Active Appearance Models," in Lecture Notes in Computer Science. vol. 1407: Springer Berlin / Heidelberg, 1998, pp. 484-498.

[12] K. Held, E. R. Kops, B. J. Krause, et al., "Markov random field segmentation of brain MR images," IEEE Trans Med Imaging., 16, pp. 878-886, 1997.

[13] Y. Y. Boykov and M.-P. Jolly, "Interactive Graph Cuts for Optimal Boundary \& Region Segmentation of Objects in N-D Images," in Eighth International Conference on Computer Vision (ICCV'01), 2001, 1, pp. 105-112.

[14] Y. Jin, C. Imielinska, A. Laine, et al., "Segmentation and Evaluation of Adipose Tissue From Whole Body MRI Scans.," in Proceedings of the Sixth International Conference on Medical Image Computing and Computer Assisted Interventions (MICCAI 2003), Montreal Canada, 2003, Part I, pp. 635-642.

[15] C. Xu and J. L. Prince, "Snakes, Shapes and Gradient Vector Flow," IEEE Transaction on Image Processing, 7, pp. 359-369, 1998.

[16] J. A. Sethian, Level Set Methods and Fast Marching Methods: Evolving interfaces in computational geometry, fluid mechanics, computer vision, and materials science. Cambridge, UK: Cambridge University Press, 1999.

[17] T. F. Chan and L. A. Vese, "Active Controus Without Edges," IEEE Transactions on Image Processing, 10, pp. 266-277, 2001.

[18] D. Mumford and J. Shah, "Boundary detection by minimizing functional," in International Conference on Computer Vision and Pattern Recognition, San Francisco, CA, USA, 1985, 1, pp. 22-26.

[19] E. Angelini, A. Laine, S. Takuma, et al., "LV volume quantification via spatio-temporal analysis of real-time 3D echocardiography," IEEE Transactions on Medical Imaging, 20, pp. 457-469, June 2001.

[20] T. Song, E. D. Angelini, B. D. Mensh, et al., "Comparison study of clinical 3D MRI brain segmentation evaluation," in Annual International Conference IEEE Engineering in Medicine and Biology Society (EMBS), San Francisco, CA, USA, 2004, pp. $1671-1674$

[21] M. Kass, A. Witkin, and D. Terzopoulos, "Snakes: Active contour models," in Proceedings of 1st International Conferences on Computer Vision, 1987, 1, pp. pp. 259-268.

[22] Q. Duan, G. Shechter, L. F. Gutierrez, et al., "Augmenting CT Cardiac Roadmaps with Segmented Streaming Ultrasound," in SPIE International Symposium, Medical Imaging 2007, San Diego, CA, 2007, 6509, pp. 65090V1-11.

[23] B. Vallet, E. Angelini, and A. Laine, "Variational segmentation framework in prolate spheroidal coordinates for 3D real-time echocardiography," in SPIE Medical Imaging Conference, San Diego, CA, USA, 2006, 6144, pp. 61444A.1-11.

[24] P. J. Hunter and B. H. Smaill, "The analysis of cardiac function: a continuum approach," Progress in Biophysics and Molecular Biology, 52, pp. 101-164, 1988.

[25] G. R. Christie, D. P. Bullivant, S. A. Blackett, et al., "Modelling and visualising the heart," Computing and Visualization in Science, 4, pp. 227-235, 2004.

[26] S. Herz, C. Ingrassia, S. Homma, et al., "Parameterization of left ventricular wall motion for detection of regional ischemia.," Annals of Biomedical Engineering, 33, pp. 912-919, 2005.

[27] P. M. Nielsen, I. J. LeGrice, B. H. Smaill, et al., "Mathematical model of geometry and fibrous structure of the heart," American Journal of Physiology - Heart and Circulation Physiology, 260, pp. H1365-H1378, 1991. 